\title{
Correction
}

\section{Correction: Weyer et al., Amplified Mechanically Gated Currents in Distinct Subsets of Myelinated Sensory Neurons following In Vivo Inflammation of Skin and Muscle}

In the article "Amplified Mechanically Gated Currents in Distinct Subsets of Myelinated Sensory Neurons following In Vivo Inflammation of Skin and Muscle" by Andy D. Weyer, Crystal L. O'Hara, and Cheryl L. Stucky, which appeared on pages 9456-9462 of the June 24, 2015 issue, several errors were discovered in the $x$-axis points for Figures 1, 3, and 4 and in the Electrophysiology and Data analysis sections in the Materials and Methods. The corrected figures and sentences are shown below.

1. In Figures $1, F$ and $G ; 3, A$ and $C$; and $4, A, B, D$, and $E$, the $x$-axis labels representing micrometers of pipette displacement were corrected to read $0.9,1.8,2.7,3.6,4.5$, and 5.4 after revisiting the authors' calculations, instead of $1.82,3.64,5.46,7.28,9.1$, and 10.92.

2. On p. 9457, left column, in the fourth paragraph of Electrophysiology, the second sentence should be: "The pipette tip was advanced in increasing displacements of $0.9 \mu \mathrm{m}$ held for $200 \mathrm{~ms}$, with 25 s given between steps to avoid sensitization/desensitization of currents."

3. On p. 9457, left column, in Data analysis, the second sentence should be: "Recordings for which the patch seal could not be maintained for at least $1.8 \mu \mathrm{m}$ of membrane indentation were discarded."

These errors do not affect any of the data interpretation or ultimate conclusions drawn by the paper. The authors sincerely regret these errors. 
A

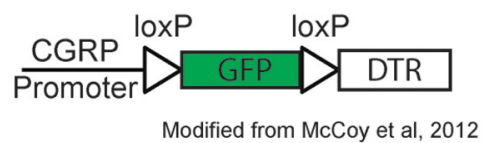

B

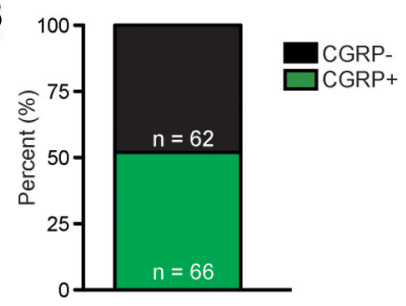

E

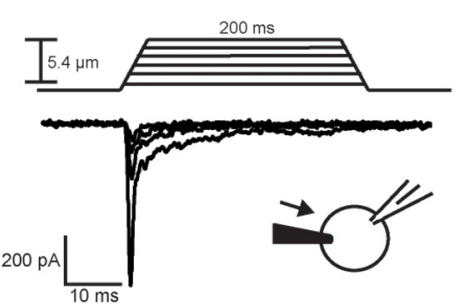

H

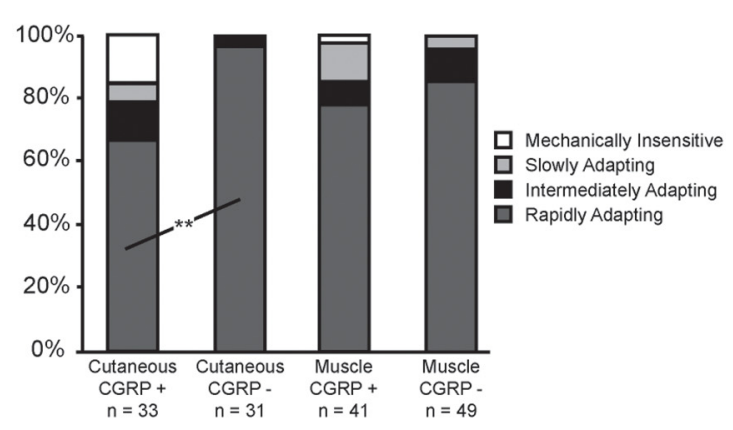

Brightfield
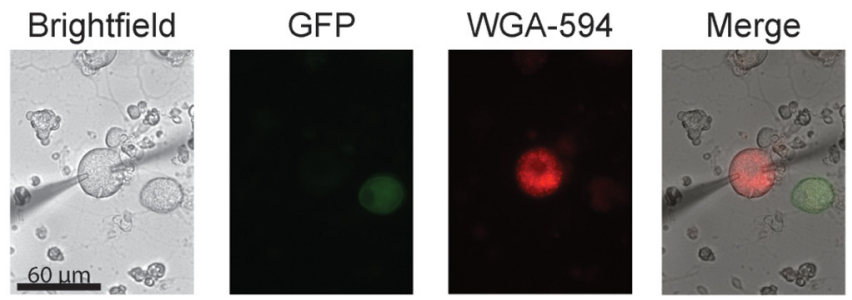

C

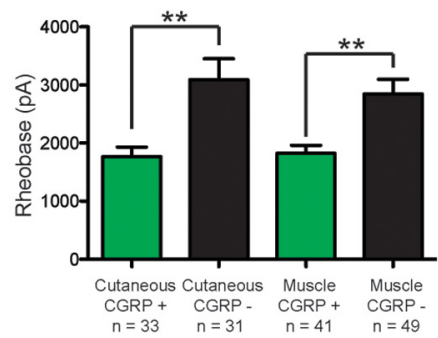

D

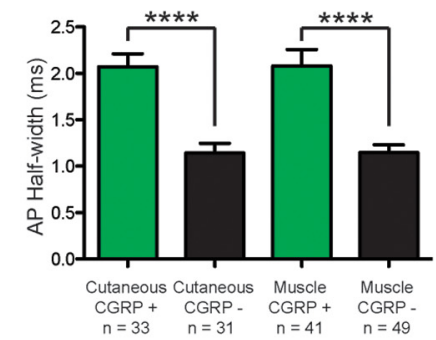

F

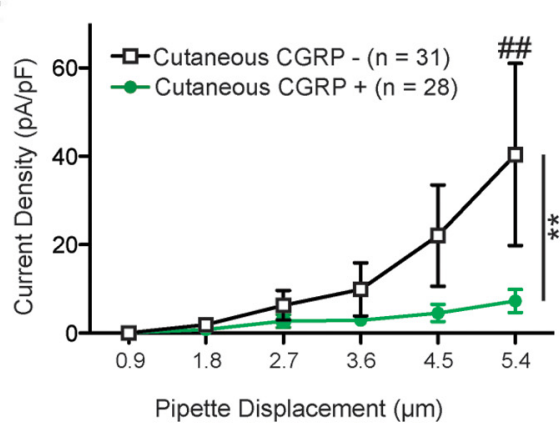

G

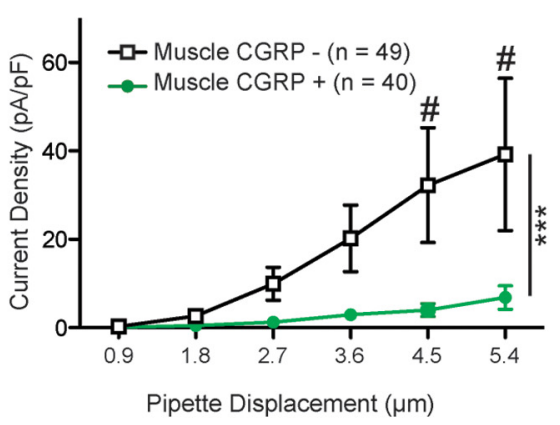

Figure 1. 
A

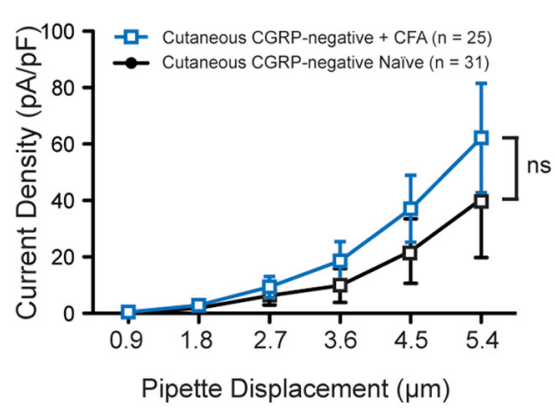

C

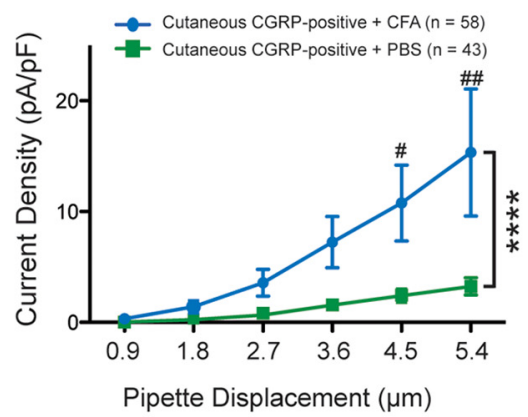

E

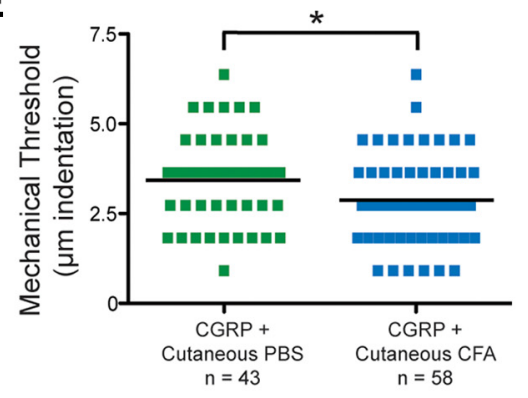

G

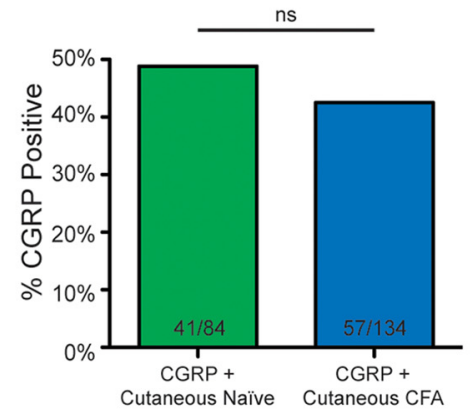

B

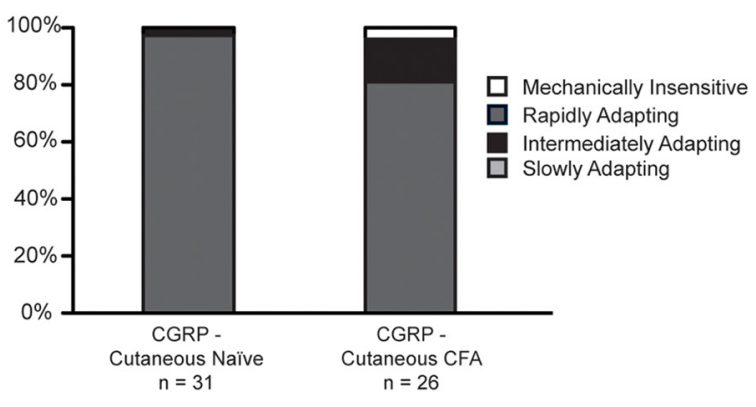

D

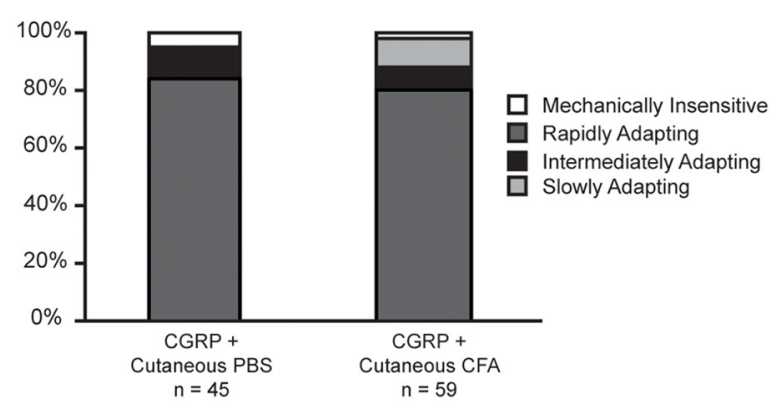

$\mathbf{F}$

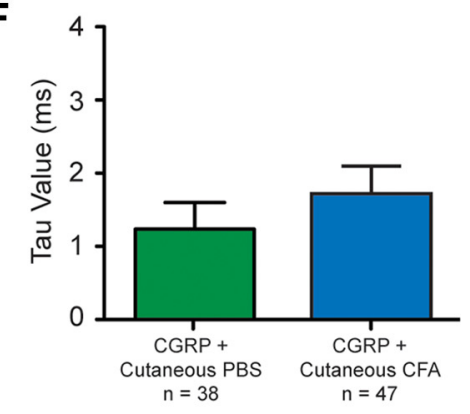

Figure 3. 
A

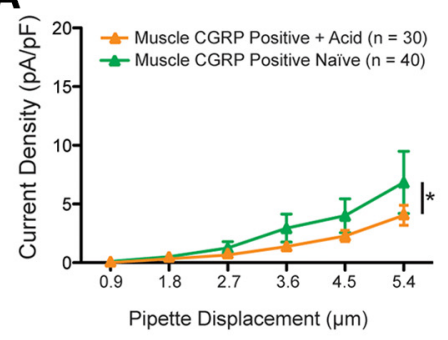

D

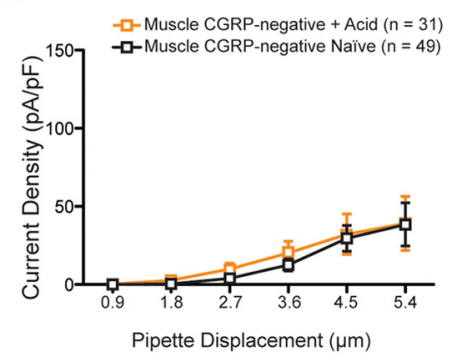

G

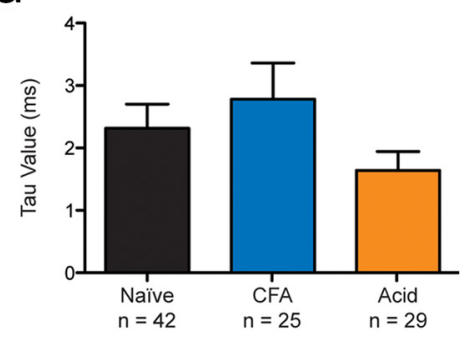

B

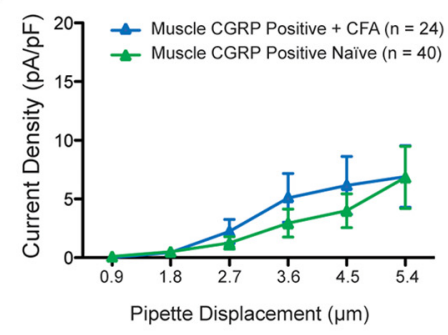

E

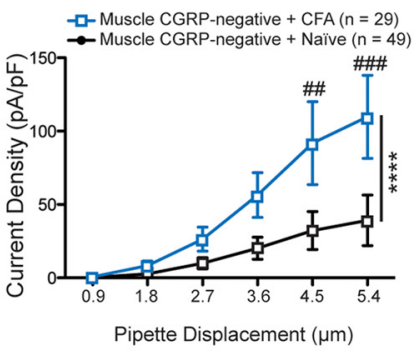

H

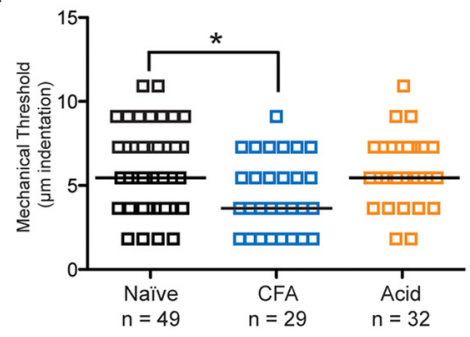

C

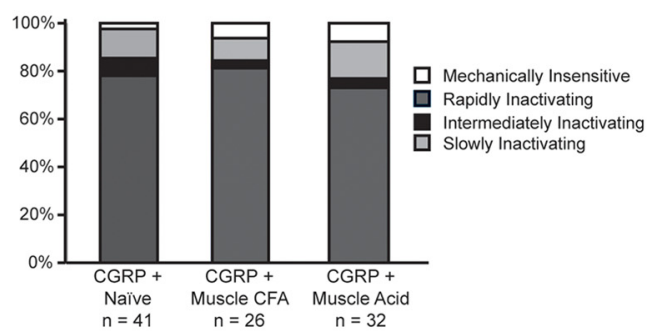

F

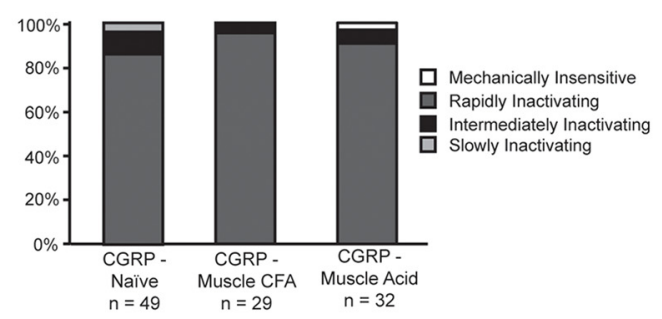

Figure 4.

DOI: 10.1523/JNEUROSCI.0232-16.2016 